\title{
El modelo de Pediatría de Atención Primaria
}

\author{
J. Ruiz-Canela Cáceres \\ CS Virgen de África. Sevilla. España.
}

\begin{abstract}
Resumen
En este trabajo comentamos las consecuencias que acarrearía un cambio de modelo sobre los pediatras y sugerimos algunos ajustes, que estimamos necesarios en el futuro para hacer dicho modelo más sostenible. Es parte de un análisis demográfico desde la perspectiva del pediatra, ya que estimamos que la carencia de pediatras es un fenómeno transitorio, aunque sujeto a decisión administrativa. Se sugieren algunas medidas organizativas, como centralizar la atención al niño en algunas zonas para evitar la dispersión y desarrollar un modelo de atención basado en pediatra y enfermera.

Sobre el modelo actual de Atención Primaria (AP) se comenta que ha estado lastrado en su desarrollo por la falta de financiación y no ha conseguido alcanzar todas sus potencialidades. Sin embargo, este modelo parece demasiado funcionarial y algunos expertos consideran necesario incentivar la autonomía de los profesionales. En el futuro inmediato, los pediatras de AP pueden jugar un papel clave, con su visión de la salud infantil más integradora y su mayor relación con las familias en la atención a problemas cada vez más complejos.

Finalmente, se considera que la coordinación entre todos los niveles, incluida la atención sociosanitaria, es imprescindible en la salud del niño y que debe ser desarrollada, se menciona la necesidad de una figura de coordinador entre niveles. Salud.

Palabras clave: Servicios de Salud del Niño. Pediatría. Calidad de la Atención de

\section{The model of Primary Care Pediatrics}

\section{Abstract}

In this paper we comment the consequences that a change in the model would have on the paediatricians and we suggest some adjustments, which we consider necessary in the future to make this model more sustainable. It is part of a demographic analysis from the perspective of the Pediatrics, because we consider that the scarcity of pediatricians in a transient phenomenon, although subdued to administration decision. Some organizational measures are suggested, like centralizing children care in some areas in order to avoid dispersion and to develop a care model based on the paediatrician and the nurse.

On the current Primary Care (PC) model we comment that it has been loaded in its development due to a lack of financing and it hasn't reached all its potentialities. Nevertheless, this model seems too civil-servant-like and some experts consider necessary to incentive the professionals' autonomy. In the near future, PC paediatricians play a key role, with
\end{abstract}

Juan Ruiz-Canela Cáceres: jruizcanela@gmail.com

El autor declara no presentar conflictos de intereses en relación con la preparación y publicación de este artículo. 
their more integrated view of child health and their better relation to the families in caring of problems more and more complex.

Finally, we consider that the coordination among all levels, included sociosanitary care is essential in children's health and it must be developed; the need of a coordinator among levels is mentioned.

Key words: Child Health Services. Pediatrics. Quality of Health Care.

En este trabajo reflexionamos sobre las consecuencias que acarraría un cambio de modelo sobre los pediatras y sugerimos algunos ajustes que estimamos necesarios en el futuro para hacerlo más sostenible en la actualidad.

\section{El análisis demográfico y los recursos humanos}

La primera cuestión que se debe abordar es el análisis demográfico desde la perspectiva del pediatra, ya que estimamos que la carencia de pediatras es un fenómeno transitorio, aunque sujeto a decisión administrativa.

La población infantil es estable en España; según datos a 1 de enero de 2009, tenemos 46661950 habitantes, esto supone un aumento de 504128 personas respecto a los datos de 1 de enero de 2008; un 12\% del total de inscritos son extranjeros. Por grupos de edad, el $15,5 \%$ de la población tiene menos de 16 años, lo que supone un incremento del $1,26 \%$ respecto al año 2007. Esto supone unos siete millones de niños menores de 16 años. En el otro extremo están los an- cianos, existe un incremento muy importante de este sector de población, que colapsa las consultas de los médicos de familia y todos los demógrafos muestran preocupación por este hecho ${ }^{1,2}$ (figura 1).

En el momento actual hay unos 8000 pediatras, y unos 5000 de ellos ejercen en Atención Primaria (AP), que tienen la responsabilidad sobre la atención de unos siete millones de niños. No hay pediatras en paro y la proporción de pediatras hospitalarios (PAH) y pediatras de AP (PAP) no se corresponde en su distribución con los problemas más prevalentes de salud del niño. Se habla de déficit de pediatras, sin embargo, como nos recuerda el estudio de Beatriz Lopez Valcárcel, dependiendo de la ratio que establezcamos puede haber déficit de pediatras para una ratio definida de niños a superávit si el número de niños que se le asigna a un pediatra es superior. Elegir un camino equivocado puede ser doloroso para los pediatras y perjudicar a la salud de los niños. Disminuir la edad de atención de manera coyuntural en zonas de déficit importante es una medida que se puede 
adoptar para asegurar una cobertura y no tan drástica, pero que debe ser siempre reversible ${ }^{3}$.

En función de los problemas de salud parece necesario que los $\mathrm{PAH}$ asuman funciones de un nivel alto de subespecialización y redireccionar las nuevas generaciones de pediatras hacia la PAP, más deficitaria.

La distribución de la población española es urbana, pues se sitúa en su mayor parte en poblaciones con más de 20000 habitantes (casi el 80\%). En poblaciones muy pequeñas no hay un número de niños suficiente y la atención por pediatras es insostenible por su escaso número. El desarrollo de las comunicaciones, permite asegurar la accesibilidad aunque se concentre a los pediatras en cabezas de co- marca con una población de niños suficiente para atender. Esta medida que introduce racionalidad va a condicionar una mejor redistribución de los PAP².

\section{Desarrollo de la especialidad de Enfermería}

Se ha publicado una Orden SAS/1730 /2010 en el Boletín Oficial del Estado de 17 de junio, por la que se aprueba y publica el programa formativo de la especialidad de Enfermería Pediátrica. Nuestro punto de vista es que debemos fomentar su desarrollo, no obstaculizarlo. Se deberían respaldar al personal de Enfermería que desde AP ha desarrollado un trabajo conjunto con los pediatras, así como contribuir al desarrollo de dicha especialidad de Enfermería. En las figuras 2 y 3 se

Figura 1. Países con mayor envejecimiento. Población $\geq 65$ años, 2000 y 2005.

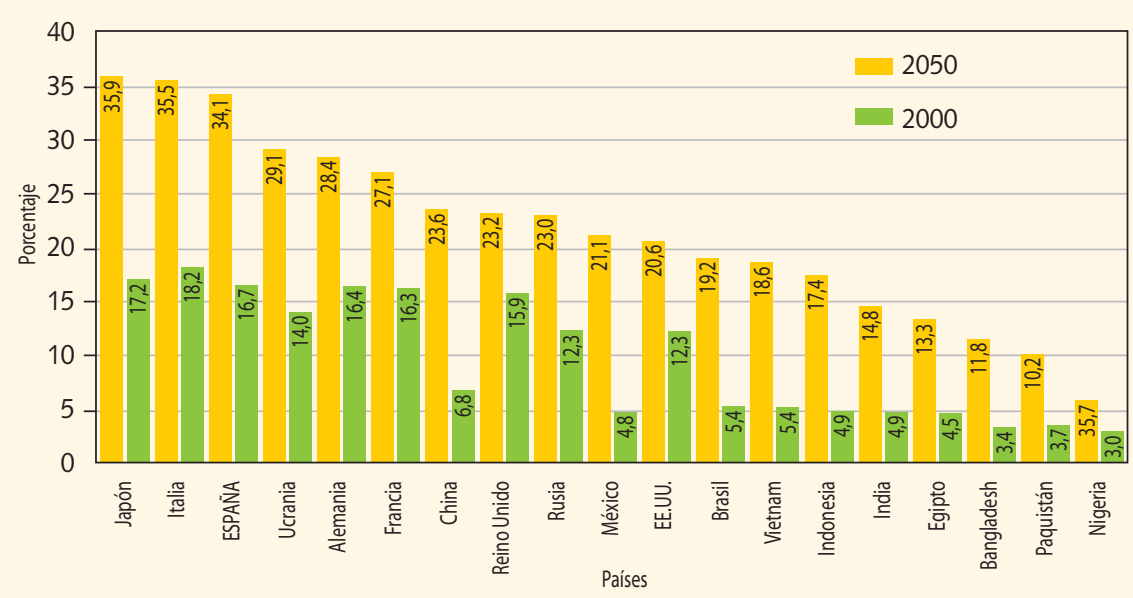


Figura 2. Revista Enfermería Pediátrica publicada en Cataluña.

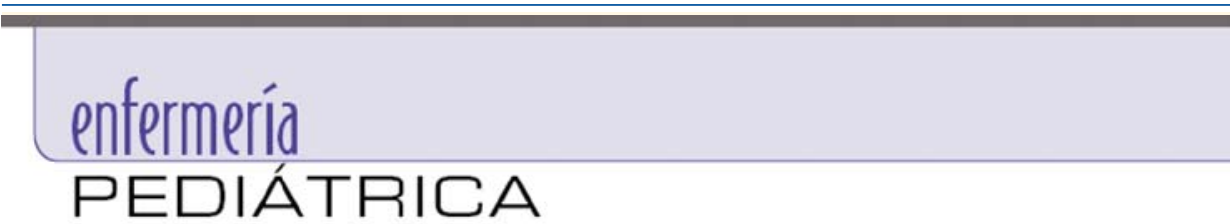

volumen $1 \cdot n^{6} 1 \cdot 2009$

EDIIORIAL

\title{
Enfermería pediátrica, ¿para qué?
}

\author{
Directores \\ Josep Bras \\ Pediatra. ABS Poblenou. Barcelona. \\ Miembro del Comite Asesor de Pediatria \\ del ICS Barceiona. \\ Cocrdinador Pediatrico de la Unidad de \\ Formación Continuada, Docencia e Investigación. \\ Barcelona \\ Francisca Jurado \\ Enfermera especializada en Pediatria. \\ Licenciada en Psicologla. \\ Miembro del Comite Asesor de Pediatria \\ del ICS-Barcelona
}

Los pediatras y enfermeros y enfermeras de pediatria de atención primaria acompanan a los ninos ( $y$ a sus familias) desde el seno materno hasta que alcanzan su autonomia personal.

La atencion primaria pediatrica incluye la promoción y la educación de la salud (individual, familiar y comunitaria), la prevención de enfermedades y accidentes, la atención a procesos agudos (diagnostico, tratamiento y educación) y el asesoramiento a las familias de niños y adolescentes con enfermedades crónicas.

El/la enfermerola de pediatria es un profesional de con-

Figura 3. Datos recopilados por Concha Sánchez Pina (Madrid) sobre implantación de Enfermería Pediátrica en el año 2010.

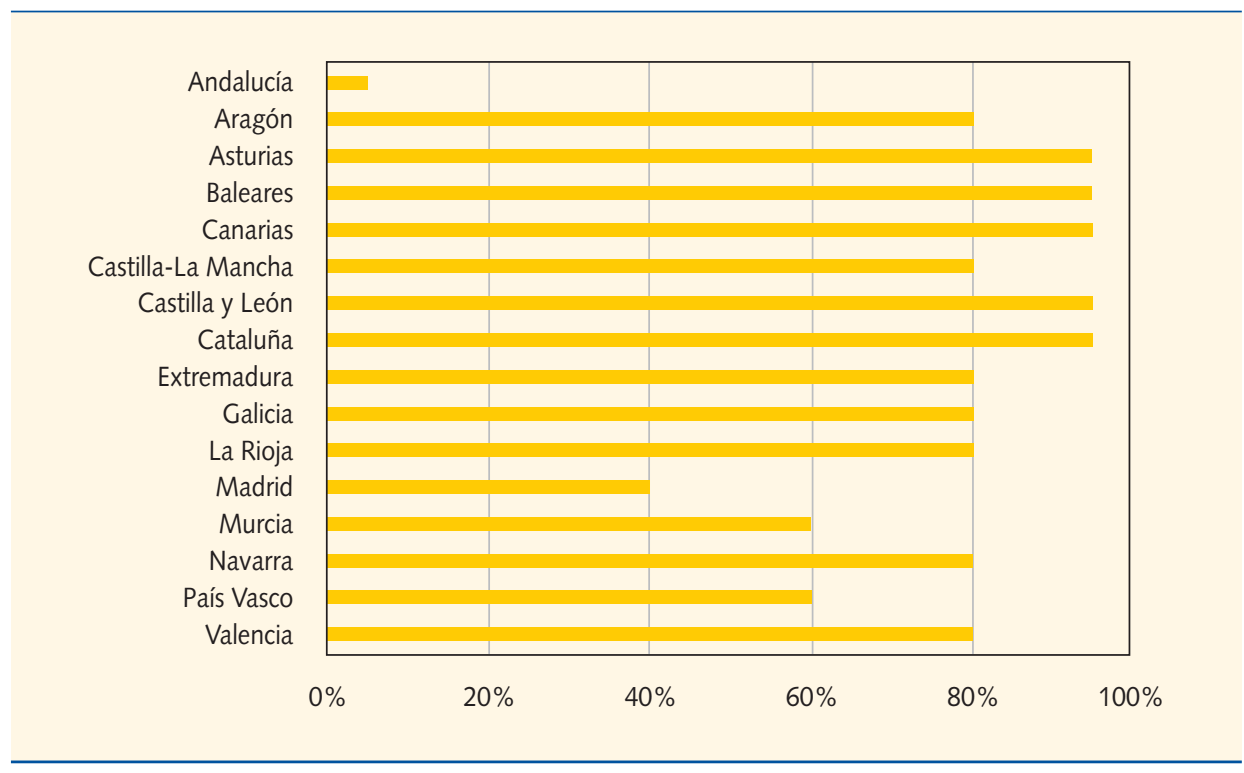


muestran datos de todas las comunidades. Se comprueba que existe Enfermería Pediátrica en la mayoría de las comunidades, y con un nivel de implantación estimable. Sin embargo, debemos matizar que no se trata de generalizar la categoría de enfermero/a especialista en el ámbito de la atención pediátrica, pues un gran número de enfermeros/as ya han adquirido dicha formación con la experiencia y el trabajo conjunto con los PAP4.6.

Un modelo asistencial que debería consolidarse para la atención al niño en el ámbito de AP es el integrado por un pediatra y un miembro de Enfermería, y que cuente con la participación activa del trabajador social en el proceso de atención directa, el apoyo personalizado del personal administrativo y la complementariedad de toda la Unidad Básica (UAP). Esta unidad básica asistencial podría concebirse como una unidad jerarquizada, pero resulta más aconsejable entenderla como una unidad interdisciplinaria que comparte pacientes y se responsabiliza de ellos de forma solidaria; entendible como una unidad integrada por profesionales que se complementan.

\section{La gestión clínica}

En un informe de la Sociedad Española de Directivos de Atención Primaria se comenta el desarrollo de la AP a lo largo de
30 años, y se constata que el modelo previsto de atención integral no se ha logrado en aspectos psicológicos ni sociales, no se ha desarrollado adecuadamente la promoción de la salud y, consecuentemente, el autocuidado y la responsabilidad de su salud por parte del ciudadano, no se ha alcanzado la capacidad de resolución que se esperaba y, menos aún, no se ha desarrollado el papel de asesor o guía del ciudadano en su itinerario por el sistema. La explicación del fracaso, según estos expertos, es imputable a la falta de financiación económica; por el contrario, el diferencial de inversión favorable al hospital ha pesado como una losa en el desarrollo de la PAP7.

Gestores y clínicos opinan también que nuestro Sistema Nacional de Salud está excesivamente funcionarizado y creen que se debe fomentar la descentralización de la gestión, hasta aproximarse a la autonomía de gestión. Los procesos contractuales (contrato-programa, contrato de gestión o similar) deberían incluir compromisos, al menos, relacionados con actividad, cartera de servicios, calidad y asignación presupuestaria. Quizá los pediatras deberíamos sacudirnos el temor al cambio y lanzarnos a crear equipos de pediatras con base asociativa, pero sobre todo implicarnos en la gestión clínica? 
La coordinación entre Pediatría de Atención Primaria y Atención Hospitalaria

M. Sánchez Jacob ${ }^{8}$ define la atención integrada en el ámbito infantil como aquella que contempla cada problema de salud desde el aspecto de prevención del problema con la promoción de los hábitos y conductas saludables relacionados con la situación, desde el aspecto asistencial de diferentes niveles $\mathrm{y}$, por último, desde la adaptación social, en aquellos problemas que puedan constituir un hándicap más o menos crónico, sin olvidar los colectivos de riesgo y los niños con necesidades asistenciales especiales.

En general, la mayoría de los niños enferman de manera aguda, en dichos casos no es muy imprescindible la integración, ya que se resuelven en un solo nivel, casi siempre en el ámbito de la PAP.

Sin embargo, en la patología crónica y dependientes, el paciente transita de un nivel de pediatra de AP a Atención Hospitalaria, y la atención a estos niños suele a menudo estar fragmentada. La atención a la patología crónica exige en su coordinación tener una visión de conjunto y una perspectiva de trabajo multidisciplinario; en mi opinión, el PAP tiene la situación privilegiada para hacerlo. También sabemos que la tecnología hospitalaria es la máxima responsable del aumento del gasto sanitario y los necesarios avances de la tecnología deben ir acompañados de una racionalidad en su distribución. Por ejemplo, las Unidades de Cirugía Cardiaca Infantil no pueden situarse en cada ciudad y debe fomentarse su ubicación en centros de referencia. Los PAP, por su cercanía, deben asumir la mayor parte del cuidado y la mejora en la tecnología debe invertirse también en el seguimiento. Esta transferencia de funciones del pediatra hospitalario al pediatra de $\mathrm{AP}$, que ya cuenta con una cartera de servicios amplia, supondrá sin duda un incremento de complejidad en su trabajo, que debe incentivarse.

De igual manera, se deben propiciar mayores funciones de la Enfermería Pediátrica. Una mayor racionalidad en la distribución de recursos contribuirá a la sostenibilidad del sistema sanitario y es un argumento que pesa cada día con más fuerza sobre el sector y las administraciones.

Hay pocos programas o procesos que tengan como objetivo la atención infantil en su conjunto y que se hayan consolidado. Las únicas excepciones son el asma y las inmunizaciones. Los hospitales pediátricos que disponen de subespecialidades suelen tener un número mayor de programas que en su mayor parte se centran en el manejo 
del niño dentro del hospital, mientras se descuidan otros problemas comunitarios, excepto el ya mencionado del asma, por el impacto que tiene en la hospitalización. Sin embargo, tenemos experiencias en nuestro país donde se ha creado una figura de coordinación en Pediatría que han impulsado la relación entre los niveles asistenciales y que han condicionado beneficios y resultados, como una mejor adecuación en la derivación, una disminución de las listas de espera y una mayor satisfacción del paciente. También se ha producido una disminución de las quejas relacionadas con las listas de espera y los retrasos en las citas, y una mejor comunicación de los test y procedimientos. Trabajar en departamentos estanco, sin conexión ni cohesión, hace que la atención a nuestros pacientes presente multitud de inconvenientes: mensajes contradictorios, duplicidad de pruebas, acciones solapadas, retrasos en la atención y un largo etcétera, que complican la asistencia y ocasionan incomodidades en los niños y sus familias. En mi opinión, la necesaria coordinación puede basarse en acuerdos entre organizaciones en modelo de redes y no impuesta de manera jerárquica ${ }^{9-11}$.

Un segundo objetivo en la coordinación para mejorar la calidad de la aten- ción es combatir la variabilidad no deseable en su atención' ${ }^{9}$. En el ámbito pediátrico hay necesidad de un sobreesfuerzo de coordinación, en mayor medida si cabe que en la medicina de adultos. Las características de los hospitales comunitarios y de referencia son muy diferentes, lo que condiciona que esa relación esté muy poco estructurada sobre la base de guías clínicas, proceso o vías clínicas, que además se realizan a espaldas de los PAP. Un objetivo fundamental de esta coordinación es favorecer la comunicación entre los profesionales de ambos niveles para mejorar la calidad asistencial, además de mejorar las relaciones entre los profesionales. Priorizar la coordinación en función de las necesidades de salud de los niños conlleva que se aborden aspectos de la atención infantojuvenil que están muy pobremente definidos; por ejemplo, algunas enfermedades mentales, el manejo de las enfermedades agudas o la intervención comunitaria. Se debería impulsar la implementación de indicadores clave que nos faciliten una idea del desarrollo de esa coordinación, tales como los sugeridos por el RAND en el mencionado análisis de Maglione ${ }^{9}$.

El desarrollo profesional continuo (la formación) es otra función que una coor- 
dinación como la sugerida debería impulsar, ya que el pediatra está aislado dentro de un equipo multidisciplinario, y precisa de formación en competencias específicas aplicada a las condiciones de su desempeño profesional. La formación MIR médico-enfermera y su rotación por PAP es una oportunidad para impulsar esta formación troncal en Pediatría y sus áreas afines, con contenidos que aborden las necesidades globales de los niños.

\section{Propuestas organizativas}

\section{Sectorización territorial}

\section{y puntos de atención}

La atención pediátrica de AP se podría organizar en función de los recursos disponibles del conjunto de zonas básicas. Se considera un ámbito territorial idóneo, por dar atención pediátrica integrada, aquel que contiene un servicio de atención pediátrica hospitalaria y las zonas básicas de referencia del hospital.

En cuanto a los puntos de atención, los equipos de AP (EAP) serán la puerta de entrada para la asistencia de los niños de 0 a 14 años. El equipo de PAP del ámbito territorial, conjuntamente con el equipo pediátrico del hospital de referencia, deberá ser capaz de resolver la prestación de la atención pediátrica primaria y la hospitalaria, con la excepción de los pro- cedimientos considerados de Pediatría terciaria. El EAP será el responsable de dar respuesta a la visita programada y a la visita espontánea (no urgente). Con el nuevo modelo, idealmente el pediatra de $E A P$, aunque pueda encontrarse asignado a un EAP determinado, debería poder atender también a otra población diferente de su zona básica ${ }^{12}$.

\section{La organización de los recursos humanos: el equipo pediátrico territorial}

El equipo pediátrico territorial conjunto de zonas básicas está conformado por los médicos y el personal de Enfermería del Servicio de Pediatría del hospital pediátrico de referencia y de los EAP del territorio que lo tienen como referente. El número de enfermeras en relación al número de pediatras de AP debería incrementarse para llegar a una relación de 1/1.

Para facilitar la organización de la atención pediátrica en determinados entornos se debería favorecer la concentración de pediatras y enfermeras de pedlatría en servicios de PAP.

\section{Desarrollo de la red hospitalaria deseable y sostenible ${ }^{13}$}

Desde el punto de vista de la organización, tomamos del modelo gallego que es necesario catalogar las prestacio- 
nes de los distintos servicios de atención pediátrica hospitalaria. Lo podemos subdividir en:

- Prestación básica: se ofertarán en todas las áreas sanitarias, en el ámbito de AP; esto debería implicar la supresión de las consultas de Pediatría general de los hospitales, por ser innecesarias y redundantes.

- Subespecializados: se debería ser muy cauteloso en su desarrollo y solo se debería permitir algunas consultas de subespecialidades pediátricas, orientadas hacia los procesos más prevalentes en la infancia: patología respiratoria, patología digestiva y patología endocrinológica.

- De referencia: se debe ofertar en un reducido número de hospitales. Su número y ubicación responderá a criterios de ordenación de recur- sos que garanticen la calidad técnica y la eficiencia de estas unidades.

\section{Desarrollo de la figura del Coordinador de Pediatría/Jefe de Servicio/Coordinador}

Nos parece operativa y deseable la figura de Coordinador Pediatra de Área en el equipo directivo de cada Gerencia de Atención Primaria, que ejerza como coordinador de la atención infantil o Coordinador/Jefe de Servicio con cierta liberación de carga asistencial para que pueda ejercer dichas funciones.

\section{Agradecimientos}

A mis compañeros de la AEPap del grupo del modelo: Juan José Morell, Ana Isabel Díaz Cirujano, Francesc Bargall, M. Ángeles Hernández, Carlos Valdivia, Gonzalo Sanz y Manel Enrubia.

\section{Bibliografía}

1. Demografía de España. Wikipedia. [Internet] [Consultado el 14/9/2010]: Disponible en http://es.wikipedia.org/wiki/Demograf\%C3\%A Da_de_Espa\%C3\%B1a.

2. Cifras de población a 1 de enero de 2007. INE [Consultado el 14/0/2010]. Disponible en www. ine.es/prodyser/pubweb/espcif/pobl08.pdf.
3. Barber Pérez P, González López-Valcárcel B. Oferta y necesidad de especialistas médicos en España 2008-2025. Madrid: Ministerio de Sanidad y Consumo; 2009. [Consultado el 14/9/ 2010]. Disponible en www.msc.es/pro fesionales/formacion/necesidadEspecialistas/ home.htm.

4. Orden SAS/1730/2010, de 17 de junio, por la que se aprueba y publica el programa formati- 
vo de la especialidad de Enfermería Pediátrica. BOE, martes 29 de junio de 2010;157:57251-76.

5. Junta Directiva de la AEPap. El modelo de atención al niño. Rev Pediatr Aten Primaria. 2005;7:549-56.

6. Ruiz-Canela J. Tribuna: Pediatría en el modelo de la AP del siglo XXI. Diario Médico. 29 de octubre de 2008.

7. Aguilera M. El modelo de Atención Primaria está agotado: una realidad o un espejismo. En: Atención Primaria de Salud: nuevos retos, nuevas soluciones. Informe de la Sociedad Española de Directivos en Atención Primaria. Madrid: Grupo Saned; 2009.

8. Sánchez Jacob M. Atención integral a la infancia: modelo biopsicosocial. Bol Soc Pediatr Asturias, Cantabria, Castilla y León. 2001;41: 322-4.

9. Mangione-Smith $\mathrm{R}$, De Cristofaro $\mathrm{AH}$, Setodji CM, Keesey J, Klein DJ, Adams JL et al. The quality of ambulatory care delivered to children in the United States. N Engl J Med. 2007;357: 1515-23.

10. Ferris $T$, Dougherty $D$, Blumenthal $D, P e-$ rrin J. A Report Card on Quality Improvement for Children's Health Care. Pediatrics. 2001;107: 143-55.

11. Cansino Campuzano A. Coordinación atención primaria especializada nuestra experiencia en Gran Canaria protocolos consensuados. BSCP Can Ped. 2006;30(2):55-60.

12. Generalitat de Catalunya. Departament de Salud. Pla d'Ordenació de l'Atenció Pediàtrica a I'atenció primària de Salut: 2007-2010. [Consultado el 14/9/2010]. Disponible en www.gen cat.cat/salut/depsalut/pdf/pediatria2007.pdf.

13. GLOBESALUD; Proyectos y acciones de salud, S.L. Modelo organizativo-funcional de la Atención Pediátrica en Galicia. Definición del Modelo. Marzo 2009 [Consultado el 14/9/ 2010]. Disponible en www.sopega.es/adjun tos/1243464976.pdf. 